\title{
Comentario a la privatización y liberación del sistema financiero
}

\author{
Carlos Briones
}

Es innegable que un sistema financiero funcionando eficientemente puede fomentar el crecimiento económico (y de esta forma contribuir al desarrollo), ya que la principal función de los intermediarios financieros es la de posibilitar la transferencia de fondos de los ahorrantes (unidades de Gasto Superavitarias) a los inversionistas (unidades de Gasto deficitarias).

Si consideramos que tanto el ahorro como el crédito pueden considerarse como unidades de consumo que resultan de las preferencias intertemporales sobre niveles de consumo de las Unidades de Gasto en relación a los flujos de ingresos esperados (Ando y Modigliani, 1963); resulta evidente la dificultad que presentaria producir el encuentro de dos agentes económicos que deseasen vender y "comprar" respectivamente el mismo número de unidades de consumo, para los mismos periodos de tiempo y asumiendo similares previsiones de contingencia en el entorno económico (evaluación y valoración del riesgo). En este sentido, resulta evidente la enorme importancia de la intermediación financiera en el proceso de coordinación de las decisiones de ahorro y de inversión de los diferentes agentes económicos.

La especialización de los intermedios financieros permite:

- Adecuar los instrumentos de crédito y ahorro a los demandantes y oferentes de fondos. De esta forma la inversión de las unidades empresariales puede ser superior a la que permitirian exclusivamente sus recursos internos o la emisión de obligaciones para un financiamiento directo adicional; por otra parte la intermediación financiera puede ele- 
var el nivel de ahorro financiero creando instrumentos (valores) que satisfagan las necesidades $y / 0$ preferencias de las unidades superavitarias. (E. Ortiz, 1980).

- Reducir el costo del enlace entre ahorrantes e inversionistas, permitiendo una reducción del riesgo y posibilitando de esta forma mejores condiciones para los prestatarios por la diversificación de las colocaciones, las economías de escala y la acumulación de experiencias en la evaluación y control de los demandantes de crédito.

- Transformar los recursos de los ahorrantes individuales, mediante un adecuado amalgamiento y una adecuada distribución previsible de los reembolsos, en fondos para sostener inversiones de largo plazo.

Sin embargo, en la realidad, los servicios de la intermediación pueden estar insuficientemente desarrollados y puede hablarse entonces de subintermediación, cuando predominan formas de ahorro no financiero; porque no existe una adecuada intermediación intertemporal que transforme la oferta de ahorros en fondos invertibles a largo plazo; porque no existe diversificación de instrumentos financieros; 0 bien porque existan unas prácticas bancarias controladas por instituciones no- financieras; etc."

En El Salvador, el sistema financiero se ha caracterizado por una creciente sub-intermediación (disminución de las operaciones pasivas y activas, represión financiera, polítización del crédito, etc.), al grado de que existe un relativo consenso sobre la necesidad de una reforma del sistema financiero, aunque no sobre las modalidades de la misma. (Ver FUSADES, 1989; CENITEC, 1989 y 1990; Banco Central de Reserva, 1990).

La forma financiera que actualmente se encuentra en proceso de implementación, tiene como ejes fundamentales la re-privatización de la banca y la disminución del papel del ESTADO en la regulación del Sistema, como instrumentos básicos para lograr la "despolitización" del sistema financiero.

En consecuencia, las reflexiones que desarrollaremos a continuación giran en torno a la adecuación de las medidas senaladas anteriormente. para lograr una mejora de la eficiencia de la intermediación bancaria.

Antes de avanzar, es necesario aclarar que estamos en total acuer-

- Igualmente puede hablarse de exceso de intermediación cuando las instituciones financieras (particularmente los bancos) subestimen los riesgos de no pago de los deudores y fragilizan el sistema por un exceso de créditos. 
do sobre la necesidad de lograr tasas de interés positivas como un medio para estimular el incremento del ahorro financiero (productivo) y también como un medio para mejorar la asignación de los recursos de inversión. Sin embargo, este objetivo (tasas reales positivas) creemos que es posible lograrlo aún en un sistema financiero nacionalizado, siempre y cuando, sea un objetivo explicito de la política monetaria y crediticia.

Entonces, el problema se centraría en establecer si la privatización y la liberalización del sistema financiero es la mejor o la única forma de promover una mayor profundización financiera, es decir, un aumento del peso del cuasi-dinero (depósitos de ahorro y depósitos a plazo) sobre el PIB. Como consecuencia de una más eficiente intermediación bancaria y además estimular simultaneamente el crecimiento del producto y del empleo.

Para responder a la anterior interrogante es necesario que definamos previamente las propiedades deseables de un sistema financiero en base a dos criterios: a) su capacidad de contribución al logro de la eficiencia asignativa; b) su contribución al logro de la mantención de la estabilidad macro-económica. En relación al primer aspecto, un sistema financiero eficiente, debe de ser capaz de permitir una reducción y una diversificación del riesgo de los depositantes (eficiencia asignativa para los recursos de las unidades de gasto superavitarias) mediante: i) una adecuada relación capitaVdepósitos; ii) una adecuada relación deuda/ capital; iii) una adecuada diversificación de las carteras de colocaciones; iv) Il(mites a la formación de conglomerados empresas-bancos; v) una propiedad bancaria suficientemente desconcentrada; y vi) una amplia gama de activos secundarios diversificados en cuanto a plazos, riesgos y retornos. (A. Solimano, 1987).

Por otra parte, otro requisito de eficiencia del sistema financiero (del lado de los inversionistas) viene dado por su capacidad de asegurar 0 apoyar (a través de la intermediación) una tasa de acumulación de capital de largo plazo, es decir, permitir o potenciar inversiones que exploten las altas tasas de retomo social de largo plazo. (Dlaz Alejandro, 1984).

Dada la generación de externalidades inherente al sistema financiero (capacidad de transformar y transmitir una crisis financiera de liquidez en crisis en el sector real); la estabilidad de tasas reales de interés la capacidad de los sistemas de información sobre los deudores, y la adecuada toma de riesgos en los préstamos; son elementos claves (y deseables) para evitar que las malas colocaciones de cartera se traduzcan en reducciones de las transacciones reales (caida de empleo y 
producción) o en costosas operaciones de rescate por otra parte del prestamista de última instancia: Banco Central.

De acuerdo a lo anterior, es claro que la mayor eficiencia del sistema no se deriva necesariamente de la privatización de los bancos ni de una liberalización rampante de la política crediticia (particularmente de las tasas de interés), sino más bien de una adecuada regulación del sistema que permita una mayor descentralización de las unidades financieras, para garantizar un grado deseable de competencia (necesaria para la diversificación de instrumentos financieros y para disminución de los diferenciales de intermediación), asi como de una política realista de tasas de interés internas que la vuelva competitivas vis-a-vis las tasas internacionales para activos financieros de similares caracteristicas.

El mayor argumento en favor de la privatización de los bancos, resulta entonces de la presunción de que un sistema bancario es más competitivo que un sistema de propiedad estatal, el argumento se basa en la idea de que el monopolio estatal (propiedad de los bancos y control de los organismos normativos) estimulan o hacen inevitable la coordinación entre los bancos, haciendo inexistente la competencia entre los bancos estatales.

Evidentemente, si entendernos la competencia como una permanente rivalidad entre los empresarios (unidades economlcas) por expandirse y ganar mayores cuotas de participaclón en los mercados, una situación como la descrita anteriormente podrla presentarse fácilmente por la existencia de un único propietario de los bancos.

Sin embargo, la posibilidad de una mínima competencia también queda abierta en un sistema bancario privado, cuando el control del sistema pasa a manos de un estrecho grupo oligopolio o bien cuando el sistema es un agregado de conglomerados empresas-bancos.

Adicionalmente, se aduce en contra de la propiedad estatal, que los administradores de las unidades financieras intermediarias, pienden interés por la evaluación adecuada de los créditos concedidos (en materia de exigencia de garantías y resguardos para cobertura del riesgo de no reembolso) y se ven desestimulados a propiciar una "absorción creativa" de las innovaciones en nuevos productos financieros, debido al rígido control del monopolio estatal y a la ausencia de estímulos vinculados a la solidez financiera de los bancos.

Este argumento, tiene bastante peso dado la conocida rigidez del proceso de toma de decisiones en las empresas públicas. Sin embargo, es más un problema de gestión y de adecuados incentivos que un pro- 
blema de propiedad, pues en la mayoria de las grandes empresas modernas el control de propiedad no se traduce necesariamente en control de la administración y los actores toman decisiones correctas o incorrectas (actuando racionalmente) dentro de los limites que permiten la estructura organización del sistema.

Evidentemente para un banco, la rentabilidad de sus operaciones depende de maximizar el diferencial entre sus ingresos directos (capacidad de colocar sus depósitos a tasas activas suficientemente remunerativas) y sus gastos financieros (intereses pagados por depósitos) y sus gastos administrativos. Por tanto, si no hay intervención directa del "monopolio" en la determinación de las colocaciones y si existe una adecuada política de tasas de interés reales, entonces un "administrador" de un banco privado y un "administrador" de un banco estatal estarian en igualdad de condiciones para maximizar los resultados de las operaciones de sus respectivas unidades financieras, diferenciándose únicamente por la capacidad bancaria, es decir, por el tamano del establecimiento y la calidad del personal directivo.

De to anterior resulta que, no es el carácter privado de los bancos lo que garantizaria una mayor eficiencia asignativa del sistema financiero, sino más bien es la adecuada re-definición de los roles de los organismos normativos del sistema (superintendencia. Banco Central y Junta Monetaria) lo que garantizaria el logro de tasas de interés reales y un grado suficiente de autonomia de parte los intermediarios, y permitiria la creación de condiciones para una competencia más libre y más acrecentada.

Finalmente, otra crítica a la propiedad estatal de los bancos, es que los acreedores de los bancos (depositantes) pierden el interés de evaluar los riesgos (de sus colocaciones) debido a que dichas instituciones cuentan con la garantia total del estado. Esto desestimula el logro de mayores niveles de eficiencia asignativa del sistema y facilita la manipulación del riesgo por parte de los intermediarios, al momento de prestar a las unidades deficitarias (demandas de crédito). Sin embargo, si se acopta plausiblemente que los compromisos de no intervención de parte del Banco Central y/o los gobiemos ante una crisis financiera de los bancos privados no son creibles (Diaz Alejandro), entonces un sistema privado regulado no es necesariamente superior a otro sistema bancario estatal regulado.

Evidentemente, si se plantea la necesidad de un sistema financiero regulado y si se acepta como plausible la organización de un sistema bancario estatal lo suficientemente descentralizado (redefinición de roles de los organismos nomativos) como para garantizar un satisfactorio 
grado de competencia, entonces la supuesta mayor eficiencia del sistema bancario privado desaparecería.

Lo anterior, no obstante, no sería prueba suficiente de la necesidad de mantener un sistema financiero/bancario estatal, dado que siempre existirla el problema de precisar el "grado óptimo de intervención", que evitarla caer en la ineficiencia asignativa.

La justificación de un sistema bancario estatal o de un sistema bancario privado adecuadamente sur-ordinado a la política económica del gobierno, sería la aceptación del derecho y del deber del gobiemo a jugar un papel activo en la dirección del proceso de acumulación y en la promoción del desarrollo (French-Davis, 1988). Entonces, la necesidad de una fuerte regulación estatal sobre el sistema bancario se derivarla de la existencia de una estrategia de desarrollo/clara y coherente. La inexistencia de esto último puede hacer innecesario el control gubernamental de los bancos y a contrario la existencia de una estrategia bien definida puede hacer imprescindible el control del sistema financiero. Esto puede ser ejemplificado con el caso de la República de Corea del Sur, en donde la subordinación de la dimensión financiera a la estrategia de desarrollo industrial condujo a que el "gobierno no sólo controlara los bancos, sino las tasas de interés del sistema bancario y los flujos de capital externo; el racionamlento del crédito de largo plazo le permitió, además, adquirir un control considerable sobre las empresas, para inducirlas a atender los requerimientos del programa exportador" (Bekerman 1986).

Si la eficiencia de asignación de los recursos es sancionada por la capacidad de competir en los mercados mundiales, el caso de Corea del Sur nos indica que una adecuada política de Intervención estatal (en la esfera real y en la esfera financiera) puede rendir muy buenos frutos en términos de desarrollo económico.

Evidentemente, lo anterior no significa que se haría de los bancos organismos Intermediarios de subvenciones o ventajas decididas por el poder público (la banca nacionalizada para ser responsable de su equilibrio de explotación debería también contar con la suficlente autonomía de gestión), significaría más bien que las administraciones de los bancos deberian "interiorizar" los objetivos del plan y abandonar la subordinación exclusiva a los crlterios unidimensionales de rentabilidad financiera; significarla también la posibilidad de acompanar al gobiemo en el desarrollo de políticas voluntaristas de desarrollo de ciertos sectores prioritarios."

Oro argumento en favor del control estatal sobre el sistema financiero, viene dado por consideraciones en tomo a la desigualdad en el 
acceso a recursos de poder económico (y poder político) entre los diferentes grupos sociales y a las consecuencias de esta desigualdad sobre la orientación de la política económica y sobre la capacidad de los grupos a defender sus intereses.

Si consideramos que el poder de un grupo social cualquiera puede definirse como la capacidad de realizar sus intereses objetivos especificos beneficiándose de la desigualdad de reacciones de los otros gnupos en relación a las suyas propias, es evidente que la reprivatización del sistema financiero se constituye en un hecho que contribuiría al reforzamiento de la desigual distribución de los recursos de poder entre los grupos sociales, a partir del momento en que dicha distribución no fuese perfectamente igualitaria.

El sistema financiero puede ser considerado un "centro de poder formalizado" (Matus, 1980) en el sentido de que tiene el monopolio estable y formal de la realización de ciertas actividades y/o decisiones, asi como también por el hecho de que su funcionamiento es codificado por un sistema de reglas que limitan el acceso y el manejo de sus recursos de poder.

En control de un "centro formalizado de poder" faculta a los grupos sociales y/o fuerzas sociales beneficiados a poder influir o contrarrestar las acciones de otros grupos y/o fuerzas sociales.

El control de crédito otorga un cierto control sobre el proceso de acumulación, la dirección de la acumulación y el tipo de acumulación en la esfera real. El control del crédito otorga a los propietarios de los bancos un relativo poder de veto sobre las demandas y/o necesidades de recursos financieros de otros empresarios y/o unidades económicas. Por otra parte la vinculación empresa (gnupos económicos)/bancos puede desnaturalizar la función de intermediación bancaria haciendo de los bancos exclusivamente "ventanillas" para colectar recursos para las empresas vinculadas con lo cual no sólo habria desigualdad sino tam-

- Evidentemente, dado el riesgo de represión financiera, el gobierno debería contar con un sistema de prospección estratégica de mercados, lo suficientemente desarrollado y calificado. Así como un sistema de información ecsnómica detallada.

Conocidos los pobres resultados de la reforma financiera de 1980, los requisitos anteriores se vuelven indispensables, ya que la ausencia de plan (y planes sectoriales) as l como la poca capacidad del gobierno para desarrollar información política económica, sólo condujo a acentuar la represión financiera y mostró que la estatización de la banca no se refleja necesariamente en una mayor aceleración del proceso de desarrollo económico. 
bién ineficiencia asignativa por baja diversificación del riesgo para los recursos de los depositantes, asi como por la baja evaluación del riesgo (o rentabilidad) de las colocaciones.

Este enorme poder es en cierta medida regulado por los organismos normativos del sistema (Banco Central, Superintendencia) pero sigue siendo de todas formas un factor de desigualdad económica.

En el caso del monopolio estatal, se podria senalar que ese enorme poder, en un régimen presidencialista, tenderia a concentrarse en manos de un sólo hombre: el Presidente de la República.

Sin embargo, si los organismos normativos son suficientemente autónomos y descentralizados y si hay una mayor transparencia y juego democrático, este poder estaria muy regulado y asi como esperamos que haga buen uso de los recursos que la colectividad pone a su disposición (PRESUPUESTO DE LA NACION) dados los controles existentes; podria esperarse que con un sistema más complejo de regulación del sistema financiero (autonomía de la superintendencia del sistema) y una política consensuada de descentralización de las unidades financieras el poder discrecional del "monopolista" estatal seria disminuldo.

¿Banco privada o banca estatal? ¿liberalización o regulación? del sistema financiero, las pruebas empiricas sobre las ventajas de cada una de las alternativas en juego no son suficientes. De hecho, como senala Diaz Alejandro (1984) sobre la experiencia de liberalización financiera del cono sur "la liberalización de tasas de interés y la relajación de los controles sobre los intermediarios financieros no estimula necesariamente la intermediación de activos de mediano y largo plazo. El florecimiento de intermediarios privados en el cono sur, incluso en la etapa del boom, se limita a un crecimiento de los depósitos y préstamos de menos de seis meses de duración... aunque al finalizar la represión financiera indudablemente se estimula cierto tipo de ahorros financieros ... el record de la inversión privada chilena durante la década de los 70 fue muy poco impresionante", o como lo sefialan Schmidt y Zcitinger (1989) a propósito de las debilidades del sistema financiero salvadoreno" ...el otorgamiento del crédito se ha decidido muchas veces en función de la influencia política del prestatario; asi mismo, las autoridades han concedido el financiamiento como una compensación indirecta por los efectos de la inestabilidad socio política". Lo anterior pareciera indicar que la alternativa seria la de construir un sistema suficientemente descentralizado, pero adecuadamente regulado.

En un sistema de este tipo la autoridad monetaria deberia establecer una estructura flexible de tasas de interés activas y pasivas fijando 
una tasa básica que guarde relación con el movimiento de índice de precios y con las tasas prevalecientes en el mercado internacional y estableciendo un margen bancario máximo entre las tasas pasivas y activas. Por otra parte las tasas básicas deberian de aumentar progresivamente en relación al plazo con el objeto de estimular a la banca a participar en el financiamiento de proyectos de largo plazo. Dado que las tasas pasivas serian positivas, podrian acordarse márgenes bancarios ligeramente inferiores a las normales, para financiar actividades consideradas prioritarias (Vrg. la Reconversión industrial). Por otra parte, la autoridad monetaria permitiria a los bancos ofrecer diferentes tasas pasivas y diferentes tasas activas dentro de un rango determinado (Vrg. tasa pasiva tijada + Margen bancario del sistema $+1 \%$ ), con esto se garantizaria la autonomía en la gestión bancaria y la competencia entre las unidades desentralizadas." La importancia de que sea el Banco Central quien defina las tasas básicas, los márgenes y los rangos de variación, es su mayor capacidad para calcular la tasa intema de retorno social y de esta forma puede garantizar que la tásas de interés cobradas no desestimulen la acumulación de largo plazo por no oscilar razonablemente alrededor de la tasa de actualización social."*

De todas formas, habiendo necesidad de reformar el sistema financiero, la agenda estaria abierta al examen de todas la alternativas. Este comentario únicamente pretende sefalar que la reforma y el estudio de ctras alternativas deberian ser objeto de una mayor reflexión y las soluciones más consensuadas. En tal sentido se apoyaria a aquellas opiniones que reclaman una nueva agenda del proceso de reforma financiera, con el objeto de estudiar alternativas en torno a las formas de propiedad y los grados de liberalización deseables.

\section{Bibliografia}

1. ANDO, A. y Modigliani, F. "The "lyfe cycle" Hypothesis of saving: Aggregate Implications and Test" American Economic Review, Marzo 1963.

2. Banco Central de Reserva "Fortalecimiento y Privatización del Sistema Financiero" Septiembre 1990.

- Un mecanismo similar es propuesto por S. de la Cuadra; S. Valdés Prieto, para facilitar la transición de un sistema estatizado a uno privado (FUSA. DES 1989). Sin embargo, no queda claro porque habiéndose logrado un grado aceptable de competencia y descentralización se debe privatizar totalmente la propiedad bancaria.

* Este caso puede ser ilustrado con las extravagantes tasas de interés registradas en Argentina, Uruguay y Chile en los 70' s. (Díaz Alejandro, 1984). 
3. Bekerman, M. "Reflexiones sobre la Experiencia Corearia". Rev. Comercio Exterior No. 8, 1986.

4. CENITEC "EI Sistema Financiero de El Salvador. Análisis y Perspectivas" Cuadernos de investigación No. 2, 1989.

5. CENITEC "La Reforma del Sistema Financiero" ¿Es oportuna la Reprivatización? Política Económica No. 2, 1990.

6. Díaz Alejandro "De la represión al colapso financiero" en "Política Económica en la Encrucijada", Colombia, 1984.

7. FUSADES "Propuesta de Modernización del Mercado de Capitales en EI Salvador", 1989.

8. French Davis, R. "Esbozo de una planteamiento neo-estructuralista" Rev. CEPAL No. 34.

9. Matus, Carlos "Planificación de Situaciones" FCE, México 1980.

10. Ortiz, E. "La Intermediación Financiera y el Desarrollo Económico", MONETARIA No. 3, 1980.

11. Solimano, A. "Liberalización Financiera Interna y Crisis: Aspectos Teóricos y consideraciones de politica Económica" en "DEUDA INTERNA Y ESTABILIDAD FINANCIERA* GEL, 1987. 